\title{
Opening the black box of machine learning in radiology: can the proximity of annotated cases be a way?
}

\author{
Giuseppe Baselli ${ }^{1}$, Marina Codari ${ }^{1,2^{*}}$ and Francesco Sardanelli,
}

\begin{abstract}
Machine learning $(M L)$ and deep learning $(D L)$ systems, currently employed in medical image analysis, are data-driven models often considered as black boxes. However, improved transparency is needed to translate automated decisionmaking to clinical practice. To this aim, we propose a strategy to open the black box by presenting to the radiologist the annotated cases (ACs) proximal to the current case (CC), making decision rationale and uncertainty more explicit. The ACs, used for training, validation, and testing in supervised methods and for validation and testing in the unsupervised ones, could be provided as support of the ML/DL tool. If the CC is localised in a classification space and proximal ACs are selected by proper metrics, the latter ones could be shown in their original form of images, enriched with annotation to radiologists, thus allowing immediate interpretation of the CC classification. Moreover, the density of ACs in the CC neighbourhood, their image saliency maps, classification confidence, demographics, and clinical information would be available to radiologists. Thus, encrypted information could be transmitted to radiologists, who will know model output (what) and salient image regions (where) enriched by ACs, providing classification rationale (why). Summarising, if a classifier is data-driven, let us make its interpretation data-driven too.
\end{abstract}

Keywords: Artificial intelligence, Decision making (computer-assisted), Diagnosis, Machine learning, Radiology

\section{Key points}

- Clinical rules and best practice require diagnosis and therapeutic decision to be transparent and clearly explained.

- Machine/deep learning offers powerful classification and decision tools, though in a hardly explained black box way.

- We propose to present the current case (CC) with training and/or validation data stored in a library of annotated cases (ACs).

- Appropriate metrics in the classification space would yield the distance between the CC and ACs.

\footnotetext{
*Correspondence: marina.codari@polimi.it; mcodari@stanford.edu

'Dipartimento di Elettronica, Informazione e Bioingegneria, Politecnico di

Milano, Via Golgi 39, 20133 Milan, Italy

2Present Address: Department of Radiology, Stanford University School of

Medicine, 300 Pasteur Dr., Stanford, CA 94305, USA

Full list of author information is available at the end of the article
}

- Proximity with similarly classified ACs would confirm high confidence; proximity with diversely classified ACs would indicate low confidence; a CC falling in an uninhabited region would indicate insufficiency of the training process.

\section{Background}

Machine learning (ML) tools and artificial neural networks, the latter nowadays progressing to deep learning (DL), are known to be data-driven models often treated as black boxes. They are currently employed in many fields of human life, including healthcare, in particular medical image analysis [1-3].

DL models are characterised by a set of parameters and hyperparameters (e.g., network topology and optimisation parameters), which allow to define a non-linear mathematical function that maps input data to target values $[4,5]$.

\section{Springer Open}

(๑) The Author(s). 2020 Open Access This article is licensed under a Creative Commons Attribution 4.0 International License, which permits use, sharing, adaptation, distribution and reproduction in any medium or format, as long as you give appropriate credit to the original author(s) and the source, provide a link to the Creative Commons licence, and indicate if changes were made. The images or other third party material in this article are included in the article's Creative Commons licence, unless indicated otherwise in a credit line to the material. If material is not included in the article's Creative Commons licence and your intended use is not permitted by statutory regulation or exceeds the permitted use, you will need to obtain permission directly from the copyright holder. To view a copy of this licence, visit http://creativecommons.org/licenses/by/4.0/. 
During model development, the massive set of parameters are iteratively tuned either to fit an annotated training set (supervised methods) or to achieve optimal clustering performances in a non-annotated one (unsupervised methods), while model hyperparameters parameters are empirically chosen applying grid or random searching strategies on the validation set. Next, the model is tested on the testing set to prove model generalizability. Therefore, DL models are the indissoluble result all the steps involved in training and validation phases that include data collection and preparation as well as augmentation and split and training and validation pipeline $[4,6]$. Indeed, even freezing model hyperparameters changing the training dataset results in completely different models.

This whole process exploits limited or no a-priori knowledge about the physical/biological behaviour of the modelled system without being explicitly programmed for a specific task [7]. However, versatility of use and ability to model complex relationship within data are reached through the design of extremely complex models.

DL data-driven approach opposes to internal modelling, which allows to define the mathematical structure of the model based on physiological a-priori knowledge and to parametrise it with few physical/physiological meaningful variables. Indeed, final DL parameters and hyperparameters do not have any meaning other than contributing to high classification performance of trained models.

Not surprisingly, the overall outcome of DL models is rather obscure, apart that it works, that is to say "the proof of the pudding is in the eating". However, we should admit that data-driven and internal models share many issues concerning the insight of the underlying mechanisms, when real clinical cases are under analysis. Indeed, the needed simplifications and approximations are transparent to few scholars. Moreover, even the most renown and established models in medicine are practically useless if the statistics of biological variability was not included.

Many issues have risen about the use of data-driven black-box classifiers in diagnostic decisions making, such as the possible reduction of physician skills, reliability of digital data, intrinsic uncertainty in medicine and need to open the DL black box [8]. Those concerns involve model real usefulness, reliability, safety and effectiveness in a clinical environment $[9,10]$.

While clinical standards may be defined to test model safety and effectiveness, model opacity represents an open issue. Indeed, the General Data Protection regulation introduced by the European Union (articles 13, 14, and 15) includes some clauses about the right for all individuals to obtain "meaningful explanation of the logic involved" when automated decision-making takes place [11]. Thus, the development of enabling technologies and/or good practices able to explain the opaque inner working of these algorithms is mandatory to comply with the important principles behind these clauses [12].

We assume that model opacity may be alleviated by enriching the DL outcomes using the information that the model derives from its training and validation dataset in a user-friendly approach, letting radiologists take their final decision with due criticism.

Paradoxically, the learning strategies of black-box $D L$ models do facilitate this task. As mentioned above, DL trained models are defined by their architecture and massive set of parameters encrypting the information of the training and validation sets. So, the training/validation sets and the trained models are assumed as being strongly and binomially linked, which bears the nontrivial consequence that also the training/validation data set should be available to users. In our vision, if data is the only prior of a black box model, this should be made transparent in the same way as physical/physiological priors must be stated for internal (alias white-box) models. Nonetheless, we illustrate a transparency principle based on highlighting annotated cases (ACs) proximal to the current case (CC) out of a library linked to the DL model. The basic requirements are as follows (i) to furnish the library of ACs (training and/or validation sets), as annex of the trained algorithm; (ii) save the coordinates of the ACs in the classification space, to be used as indexing within the library; (iii) to define a metric in the classification space permitting to univocally define the proximity of ACs to the CC.

In this article, we describe our approach focusing on a specific DL model, namely a convolutional multi-layer neural networks used to perform a binary classification task.

\section{$M L / D L$ models in radiology}

In the last years, several publications have shown the potential of ML/DL applications in medical imaging $[5,13]$. The concern is what to do with classifications performed by trained ML/DL models, since they assume that clinical tasks can be solved using sharp decision boundaries (what and where), though without providing intelligible explanations (why). Also from a clinical point of view, the threshold approach and the hypothetico-deductive model have shown several limitations, especially in primary care due to the low prevalence of specific diseases and the extent and poor differentiation of the diagnostic problem space [14]. On the contrary, searching problem space by inductive gathering and triggered routine has emerged as diagnostic strategy for generalist settings [15].

What would skilled radiologists do in the case of diagnostic uncertainty about the CC they have on the screen? Simple, they would search into digital atlases or textbooks cases like the specific one and seek information about the classification confidence of reference cases. Only if they 
found good similarity and good classification confidence, they would accept the classification proposed by the external source, though with the reported degree of uncertainty. In this light, we propose a DL model outcome inspection strategy that mimics radiologist's behaviour in a real case scenario. Currently, when complex cases are analysed using DL systems, heatmaps are compared with ground truth annotations to allow the radiologist to trust the black box systems. Figure 1 shows an example of breast arterial calcification (BAC) detection performed using a convolutional neural network $(\mathrm{CNN})$. In the heat map, only the BAC area was above threshold. Manual segmentation (Fig. 1b) is shown for explanatory reasons but was not used in the CNN training: only image level labels (present/absent) were used as ground truth. Note that even after delimiting the BAC area (Fig. 1b), e.g., by the heat map (Fig. 1c), the BAC is hardly recognised by a naif eye. Conversely, the support of the manual segmentation by an expert annotator (Fig. 1d) immediately highlights the searched structure, when back to the original. In our hypothesis, when analysing a CC with no annotation, a surrogate support to decision can be given by similar ACs. Heatmaps are a useful tool to understand which part of the image guided the DL model to its decision but does not provide information about the reason behind it. To better understand the link between that part of the image and the classification outcome, the radiologist must compare it to the ground truth annotation (if available).

However, ground truth annotations are not available while looking at the CC. To give higher support to the final radiologist decision, adding fuzzy or probabilistic information and reference images or cases, should be not difficult. Those solutions could be studied, implemented and validated, in a near future.

\section{ML/DL uncertainty made explicit: DL example}

Systems based on ML/DL neural networks are complex models composed by a massive number or nodes staked

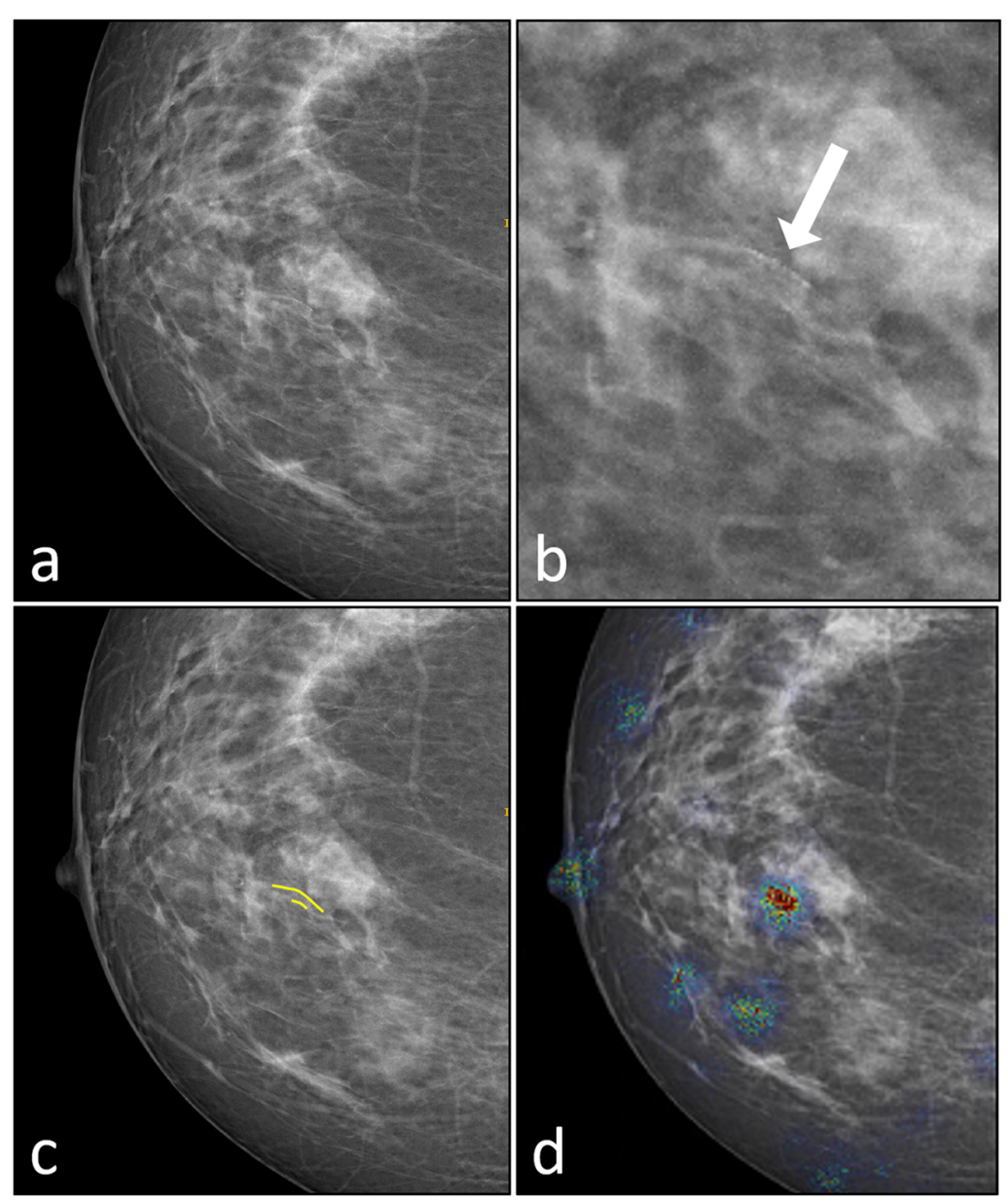

Fig. 1 Breast arterial calcifications (BAC) detection by convolutional neural network (CNN). a Original image (positive to BAC presence). b Detail including the unsegmented BAC (white arrow). $\mathbf{c}$ Heat map provided by the CNN. d Annotated image (BAC in yellow). The heat map (c) has the reduced resolution of images input to the $\mathrm{CNN}$ 
in layers [4]. To have model uncertainty made explicit, a trivial idea can be drawn by observing the classification space. For instance, in DL, this operation can be done by observing the outputs $f_{k}^{[L-1]}$ of the last hidden layer, namely the features selected by the previous deeper layers while processing a specific case. The elements of this space do next enter the summation of the output node and, through the nonlinear activation function, provide the sharp classification. In the sake of clarity, the simplest binary classification (either negative or positive), is exemplified in Fig. 2.

Indeed, elements $f_{k}^{[L-1]}$ are abstract features (alias, meta-features), which result from the passage through many layers that non-linearly combine the meaningful input features. However, they have two important characteristics: (a) validation has recognised them as major determinants of the final decision; (b) they can be put in the classification space of features and their proximity can be assessed by specific metrics, carefully selected among those available in the literature [16]. So, the examined case will be a point in this space. Even more importantly, each AC included in the library will find a precise position (fixed and recorded at the end of training or during validation) and those close to the addressed case could be rapidly retrieved through a look-up table. A theoretical example of a CC surrounded by the relevant cluster of libraries ACs is shown in Fig. 3.

\section{The radiologist entering the black box}

The first consequence of the presented approach is that the radiologist would be provided by the pertinent ACs (as by old image atlases).

The second consequence is that the proximal ACs should provide the original images and also ancillary information, such as annotation masks, annotation agreement (alias, human confidence), validation confidence (alias DL confidence), heatmaps localising image regions influencing the classification, subject's demographics, and clinical profile. The third one is that the distance of the $\mathrm{N}$ closest ACs can quantify the density of the library in the region where the current case has fallen, which implies the robustness of training and/or validation specific to the CC.

Possible instances are shown in Fig. 4: (a) the CC falls into a crowded region with high levels of consensus, which would support the automated classification and also explain it by the CC similarity to homogenous ACs; (b) the CC falls into an uninhabited region, which would highlight a lack of training and/or validation cases similar to the $\mathrm{CC}$; (c) the CC falls into a crowded area, yet with differently classified ACs, most likely in a boundary region with low confidence scores, which uncertainty can be legitimately transferred to the CC classification.

\section{Transparency and communication barriers}

It is worth emphasising that the annotation process exploited for training, validation, and testing in ML/DL

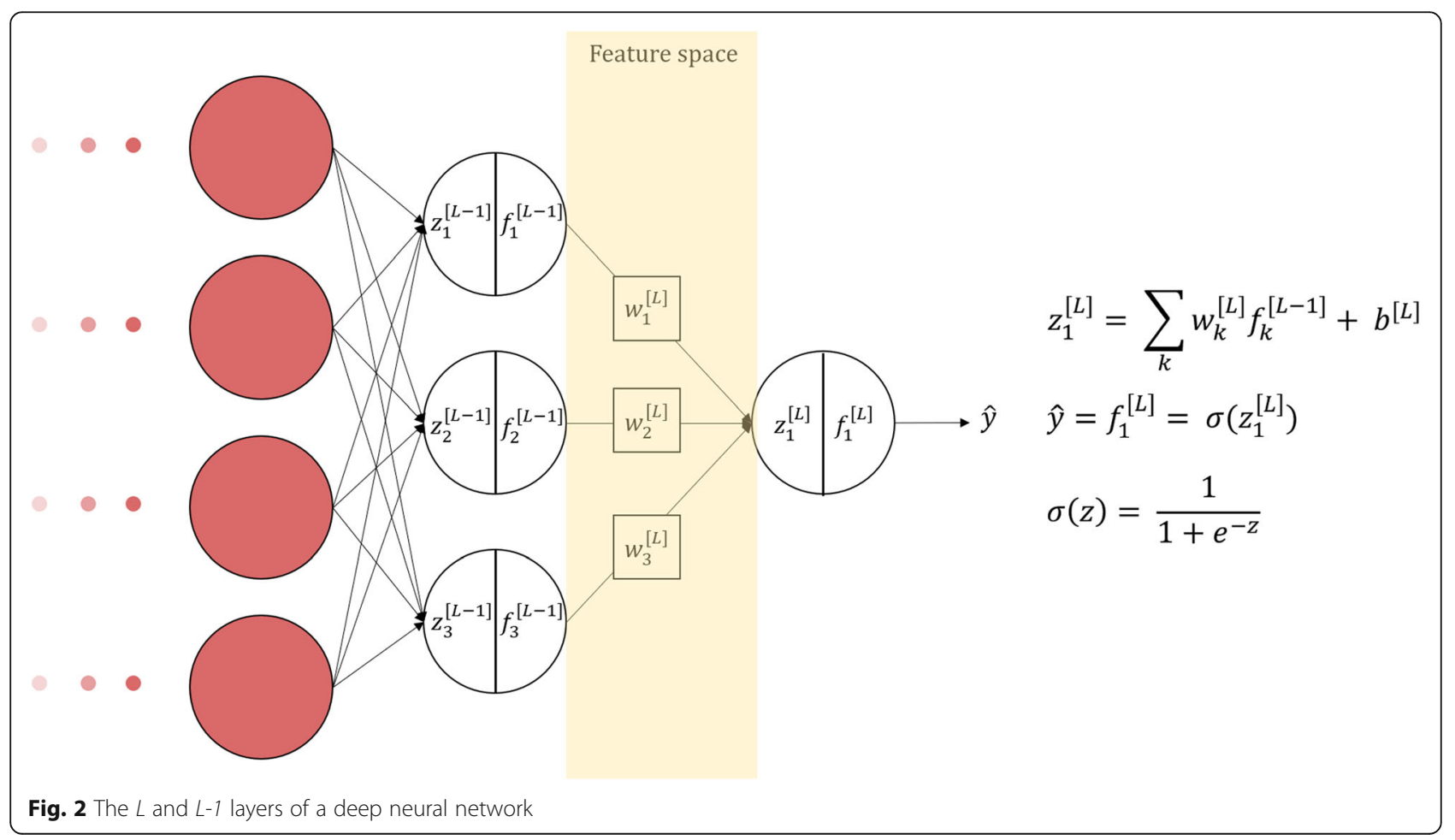




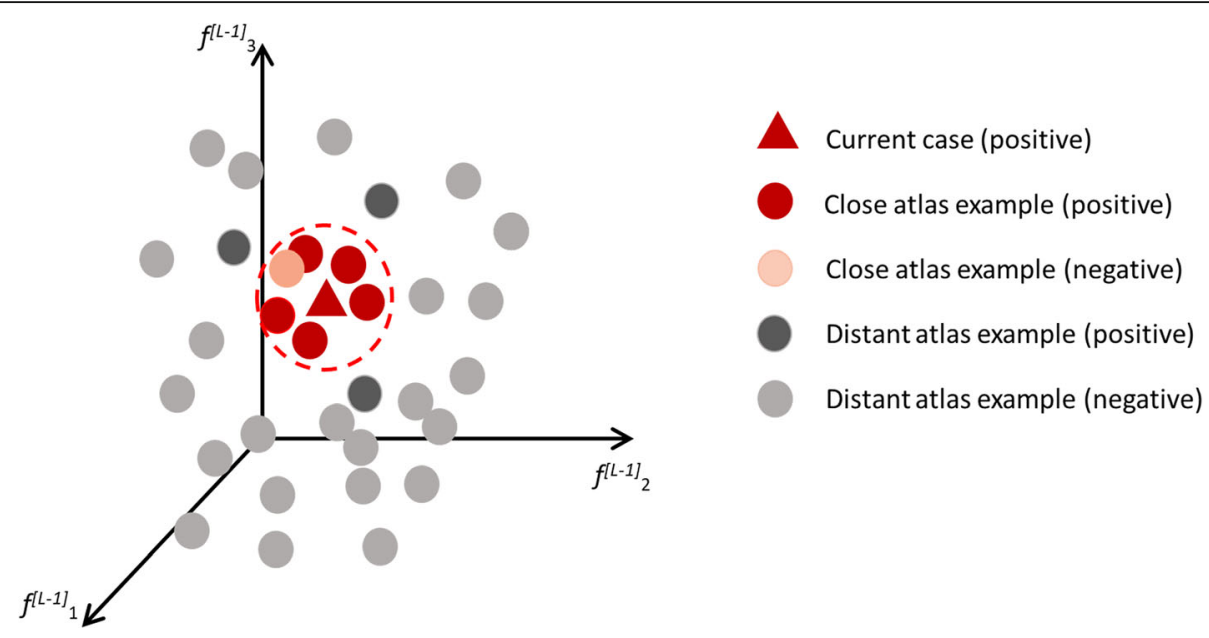

Fig. 3 The current case (red triangle) is positioned in the output feature space of L-1 layer. A neighbour region (red dashed circle) is fixed and the included training/validation annotated cases (red circles) are considered to provide reference images, classification confidence and ancillary information. Other cases outside the neighbourhood are represented as grey circles

models implies that significant clinical knowledge and rating efforts were exploited by the model developers and are ultimately encrypted inside the trained model parameters. However, as shown in Fig. 5a, this is not transmitted to the clinician in charge to the CC, who must rely on its own experience in order to justify the model prediction. Hence, a communication barrier is cast, even if the whole process from development to application subtends common clinical knowledge and classification rule consensus. Conversely, the abovementioned information is better conveyed by means of ACs from the library. The part of library relevant to the $C C$ is time to time activated based on a proximity concept. Hence, the user radiologist will benefit not only of the classification (what) and localisation capabilities (where) of the model, but also will have reference cases permitting to explain the decision (why) and assess its confidence (Fig. 5b). Furthermore, this process would help in detecting cases poorly addressed by the model, thus permitting to give feedbacks to developers, and to allow those feedbacks to be collected, verified and applied for improved model versions prior to being certified and delivered to the clinical community as a new improved release. Medicine has been often improved via empirical observations shared to the clinical community. Also, ideas for new research projects frequently arise from empirical, anecdotal observations. A black-box application of DL approaches could interrupt this virtuous-loop. Our hypothesis may facilitate comprehension of the developers' view to users (feedforward) as well as give back to developers the users observations (feedback). Nothing new, as is in many arts and in medicine.

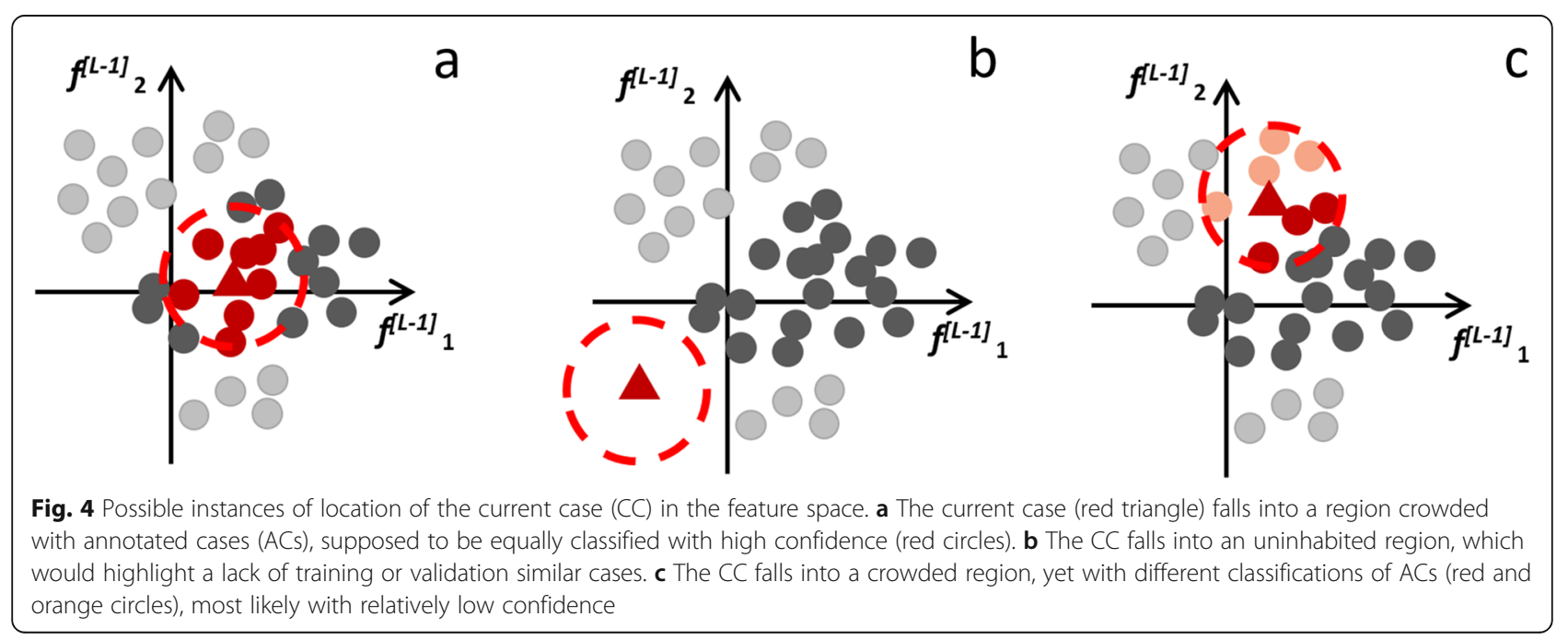




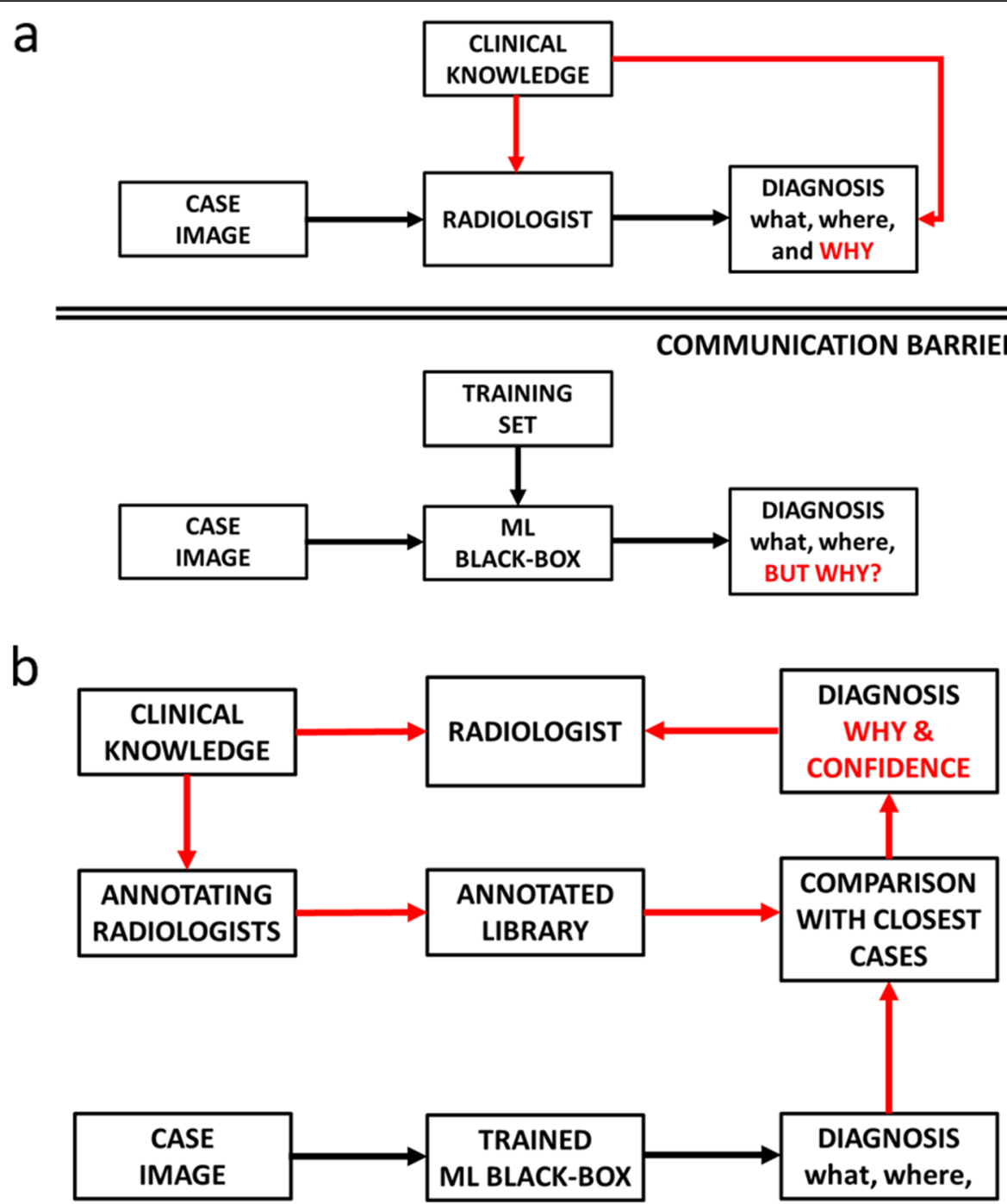

Fig. 5 Schemes of the diagnostic process aided by machine learning tools to show process differences with (a) and without (b) communication barriers. The second option allows the clinician to retrieve information about classification results (what), object localisation (where), and added information on the decision-making process (why) derived from the annotated library

Additional information provided by our solution may cause a reporting time increment. However, a close inspection of similar cases should not be done on regular basis. It should be performed mainly for critical cases and/or in order to pinpoint systematic classification flaws and for DL algorithm debugging (e.g., to enrich a class poorly represented in the training and validation sets). Moreover, more information about system decision may be provided on demand when needed.

Conversely, we foresee that the most practical outcome to clinical decision support would be to provide objective and well-explained indexes of classification confidence specific to the $\mathrm{CC}$ such as the density of the proximal classification space with similar cases. We believe that this approach will provide a significant added value to existing solutions allowing a more tailored analysis of DL outcomes compared to the indexes of the classifier performance, which give overall statistics.

\section{Conclusions}

We are currently impressed by the emerging role of ML/ DL in medicine and radiology. More and more, computer algorithms are shown to outperform radiologists, exploiting curiosity and fears of downsizing of professional roles. However, the patients' interest is not to know whether a ML/DL tool is better than a physician but if a radiologist with an $M L / D L$ aid is better than the same radiologists without.

The way to open the back box we presented here can favour an interactive cooperation between radiologists and automated systems, soliciting the radiologists' (biological!) neural networks to integrate their previous clinical experience 
by visualising well-labelled cases that the system has classified as proximal to the $\mathrm{CC}$, so allowing for a critical assessment of the performance of the automatic tool. Moreover, it may serve as a valuable tool to test the generalizability of the proposed model during development and certification processes. This perspective offers a novel paradigm of proximity for $M L / D L$ transparency. However, we did not tackle problems such as the dimensional reduction of the classification space to few weighty meta-features and the choice of the most effective metrics within. We hope that these non-trivial methodological problems might solicit brilliant minds in the field to experiment the best implementation ways.

\section{Abbreviations}

AC: Annotated case; BAC: Breast arterial calcification; CC: Current case;

CNN: Convolutional neural network; DL: Deep learning; ML: Machine learning

\section{Acknowledgements}

This study was supported by Ricerca Corrente funding from Italian Ministry of Health to IRCCS Policlinico San Donato.

\section{Authors' contributions}

All three authors participated in article design and drafting. All authors approve the final version of the manuscript.

\section{Funding}

None to be declared.

\section{Availability of data and materials}

Not applicable

\section{Ethics approval and consent to participate}

Not applicable

\section{Consent for publication}

Not applicable

\section{Competing interests}

The G.B. and M.C. declare that they have no competing interests related to the proposed study. F. S. is the Editor-in-Chief of European Radiology Experimental; for this reason, he was not involved in any way in the revision/ decision process, which was completely managed by the Deputy Editor, Dr. Akos Varga-Szemes (Medical University of South Carolina, Charleston, SC, USA).

\section{Author details}

'Dipartimento di Elettronica, Informazione e Bioingegneria, Politecnico di Milano, Via Golgi 39, 20133 Milan, Italy. ${ }^{2}$ Present Address: Department of Radiology, Stanford University School of Medicine, 300 Pasteur Dr., Stanford CA 94305, USA. ${ }^{3}$ Unit of Radiology, IRCCS Policlinico San Donato, Via Morandi 30, San Donato Milanese 20097, Italy. ${ }^{4}$ Department of Biomedical Sciences for Health, Università degli Studi di Milano, Via Morandi 30, San Donato Milanese 20097, Italy.

Received: 19 December 2019 Accepted: 1 April 2020

Published online: 05 May 2020

\section{References}

1. The Lancet Respiratory Medicine (2018) Opening the black box of machine learning. Lancet Respir Med 6:801 https://doi.org/10.1016/S22132600(18)30425-9

2. Litjens $G$, Kooi T, Bejnordi BE et al (2017) A survey on deep learning in medical image analysis. Med Image Anal 42:60-88 https://doi.org/10.1016/j. media.2017.07.005

3. Pesapane F, Codari M, Sardanelli F (2018) Artificial intelligence in medical imaging: threat or opportunity? Radiologists again at the forefront of innovation in medicine. Eur Radiol Exp 2:35-45 https://doi.org/10.1186/ s41747-018-0061-6
4. Chartrand G, Cheng PM, Vorontsov E et al (2017) Deep learning: a primer for radiologists. Radiographics 37:2113-2131 https://doi.org/10.1148/rg. 2017170077

5. Erickson BJ, Korfiati P, Zeynettin A, Kline TL (2017) Machine learning for medical imaging. Radiographics 37:505-515 https://doi.org/10.1155/2015/ 825267

6. Willemink MJ, Koszek WA, Hardell C et al (2020) Preparing medical imaging data for machine learning. Radiology 192224 https://doi.org/10.1148/radiol. 2020192224

7. Krause J, Perer A, Ng K (2016) Interacting with predictions: visual inspection of black-box machine learning models.. In: Proceedings of the $2016 \mathrm{CHI}$ Conference on Human Factors in Computing Systems - CHI '16. ACM Press, New York, New York, USA, pp 5686-5697 https://doi.org/10.1145/2858036. 2858529

8. Cabitza F, Rasoini R, Gensini GF (2017) Unintended consequences of machine learning in medicine. JAMA 318:517 https://doi.org/10.1001/jama. 2017.7797

9. Hosny A, Parmar C, Quackenbush J, Schwartz LH, Aerts HJWL (2018) Artificial intelligence in radiology. Nat Rev Cancer 18:500-510 https://doi.org/10. 1038/s41568-018-0016-5

10. Maddox TM, Rumsfeld JS, Payne PRO (2019) Questions for artificial intelligence in health care. JAMA 321:31 https://doi.org/10.1001/jama.2018. 18932

11. EUR-Lex (2016) Regulation (EU) 2016/679 of the European Parliament and of the Council of 27 April 2016 on the protection of natural persons with regard to the processing of personal data and on the free movement of such data, and repealing Directive 95/46/EC (General Da. https://eur-lex. europa.eu/legal-content/EN/TXT/?uri=CELEX:32016R0679.

12. Guidotti R, Monreale A, Ruggieri S et al (2018) A survey of methods for explaining black box models. ACM Comput Surv 51:1-42 https://doi.org/10. $1145 / 3236009$

13. Shen D, Wu G, Suk H (2017) Deep learning in medical image analysis. Annu Rev Biomed Eng 19:221-248 https://doi.org/10.1146/annurev-bioeng071516-044442

14. Green LA, Fryer GE Jr, Yawn BP, Lanier D, Dovey SM (2001) The ecology of medical care revisited. N Engl J Med 344:2021-2025 https://doi.org/10.1056/ NEJM200106283442611

15. Donner-Banzhoff N (2018) Solving the diagnostic challenge: a patientcentered approach. Ann Fam Med 16:353-358 https://doi.org/10.1370/afm. 2264

16. Böhm C, Berchtold S, Keim DA (2001) Searching in high-dimensional spaces: index structures for improving the performance of multimedia databases. ACM Comput Surv 33:322-373 https://doi.org/10.1145/502807.502809

\section{Publisher's Note}

Springer Nature remains neutral with regard to jurisdictional claims in published maps and institutional affiliations.

\section{Submit your manuscript to a SpringerOpen ${ }^{\circ}$ journal and benefit from:}

- Convenient online submission

- Rigorous peer review

- Open access: articles freely available online

- High visibility within the field

- Retaining the copyright to your article

Submit your next manuscript at $\boldsymbol{\nabla}$ springeropen.com 